\title{
Idebenone increases mitochondrial complex I activity in fibroblasts from LHON patients while producing contradictory effects on respiration
}

\author{
Claire Angebault ${ }^{1,2,3}$, Naïg Gueguen 1,2,3,4, Valérie Desquiret-Dumas ${ }^{1,2,3,4}$, Arnaud Chevrollier ${ }^{1,2,3,4}$, Virginie Guillet ${ }^{3}$, \\ Christophe Verny ${ }^{1,2,3,5}$, Julien Cassereau ${ }^{1,2,3,5}$, Marc Ferre ${ }^{1,2,3,4}$, Dan Milea ${ }^{1,2,3,6}$, Patrizia Amati-Bonneau ${ }^{1,2,3,4}$, \\ Dominique Bonneau ${ }^{1,2,3,4}$, Vincent Procaccio ${ }^{1,2,3,4}$, Pascal Reynier ${ }^{1,2,3,4,7^{*}}$ and Dominique Loiseau $u^{1,2,3,4}$
}

\begin{abstract}
Background: Leber's hereditary optic neuropathy (LHON) is caused by mutations in the complex I subunits of the respiratory chain. Although patients have been treated with idebenone since 1992, the efficacy of the drug is still a matter of debate.

Methods: We evaluated the effect of idebenone in fibroblasts from LHON patients using enzymatic and polarographic measurements.

Results: Complex I activity was $42 \%$ greater in treated fibroblasts compared to controls $(p=0.002)$. Despite this complex I activity improvement, the effects on mitochondrial respiration were contradictory, leading to impairment in some cases and stimulation in others.

Conclusion: These results indicate that idebenone is able to compensate the complex I deficiency in LHON patient cells with variable effects on respiration, indicating that the patients might not be equally likely to benefit from the treatment.
\end{abstract}

\section{Background}

Leber's hereditary optic neuropathy (LHON, OMIM \#535000), the most frequent mitochondrial DNA-related disorder, is characterized by maternal inheritance and by incomplete penetrance with male predominance $[1,2]$. The age of onset is highly variable but typically situated between 15 and 30 years. The acute or subacute onset of the optic neuropathy, due to the specific degenerescence of retinal ganglion cells, generally begins in one eye and affects the other one within a few weeks or months. Three main mitochondrial DNA point mutations (m.11778 G > A in the ND4 gene, m.3460 G > A in the ND1 gene, and m.14484 T > C in the ND6 gene) are responsible for the disease in $95 \%$ of patients $[1,2]$. These mutations affect the protein subunits of complex I of the mitochondrial respiratory chain (NADH dehydrogenase). Fibroblasts from patients carrying one of the

\footnotetext{
* Correspondence: pareynier@chu-angers.fr

${ }^{1}$ INSERM U771, Angers 49000, France

Full list of author information is available at the end of the article
}

three mutations have on average $61 \%$ residual complex I activity, associated with defective OXPHOS efficiency and impaired ATP synthesis [3].

Idebenone (2,3-dimethoxy-5-methy-6-(10-hydroxy)decyl-1,4-benzoquinone), an analog of coenzyme Q, facilitates electron transfer along the respiratory chain. The beneficial effects of this molecule remain controversial since some reports have claimed it leads to faster visual recovery [4-6], whereas other authors have found that idebenone treatment did not alter the course of the disease [7]. A recent randomized controlled trial in 85 patients with LHON showed that patients with discordant visual acuities were the most likely to benefit from idebenone treatment [8]. Another recent retrospective clinical study in 103 LHON patients showed that early and prolonged idebenone treatment may improve significantly the frequency of visual recovery [9] but, within the treated group, only a proportion of patients was shown to respond to the treatment. To date, the biological effects of idebenone

\section{Ciomed Central}


on pathological models have received little attention, although idebenone is often administered in cases of LHON. In order to better understand the biological bases of these variable impacts of idebenone in LHON patients, we tested the effects of idebenone on complex I activity and mitochondrial respiration in fibroblasts from patients harbouring one of the three main mutations associated with the disease.

\section{Methods}

\section{Ethical approval}

This work was approved by the Ethical Committee of the University Hospital of Angers ( $\left.\mathrm{N}^{\circ} 2011 / 39\right)$.

\section{Patients}

Fibroblasts were cultured from skin biopsies taken after obtaining informed consent from nine patients (eight men and one woman), each carrying one of the three mutations associated with LHON, and three controls. The LHON mutations and haplotyping were determined in our diagnostics laboratory. The heteroplasmic levels were measured at the same cellular passage numbers as those used for the experiments (Table 1).

\section{Cell culture}

The cells were cultivated in 2/3 Dulbecco's minimum essential medium (Gibco, Cergy-Pontoise, France) supplemented with 1/3 Amniomax (Gibco, Cergy-Pontoise, France) and 10\% foetal calf serum (PAA, Pasching, Austria). All experiments were conducted on cells at less than 20 passages to avoid bias due to cell senescence. Ethanol (used as vehicle) or $10 \mu \mathrm{M}$ idebenone (Santhera Pharmaceutical, Liestal, Switzerland) was added $24 \mathrm{~h}$ before each experiment. At this concentration, idebenone has been found to be effective as an antioxidant [10] and capable of stimulating the mitochondrial respiratory chain [11]. Unfortunately, we were not able to perform oxygen consumption in patients 7,8 and 9 .

\section{Enzymatic activities}

The activity of the mitochondrial respiratory chain complexes was measured on cell homogenates in a cell buffer (250 mM saccharose, $20 \mathrm{mM}$ tris[hydroxymethyl] aminomethane, $2 \mathrm{mM}$ EGTA, $1 \mathrm{mg} / \mathrm{mL}$ bovine serum albumin, $\mathrm{pH} 7.2$ ), at $37^{\circ} \mathrm{C}$ using a Beckman DU 640 spectrophotometer (Beckman Coulter, Brea, CA, USA). The cellular protein content was determined with the Bicinchoninic assay kit (Uptima, Interchim, Montluçon, France) using bovine serum albumin as standard. Complex I (NADH ubiquinone reductase, EC 1.6.5.3) activity was measured according to a procedure described elsewhere [12] and adapted using DCPIP to avoid the inhibition of complex I activity by decylubiquinol [13]. Cells were disrupted by two freezing-thawing cycles, washed, centrifuged for one minute at $16000 \mathrm{~g}$, re-suspended in the cell buffer $\left(50 \mu \mathrm{L} / 10^{6}\right.$ cells), and sonicated $(6 \times 5 \mathrm{~s})$ on ice. Complex I activity was immediately assayed on cell lysate $\left(0.25 \times 10^{6}\right.$ cells $)$ in the $\mathrm{KH}_{2} \mathrm{PO}_{4}$ buffer $(80$ $\mathrm{mM}, \mathrm{pH} 7.4$ ), containing $1 \mathrm{mM} \mathrm{KCN}, 2 \mathrm{mM} \mathrm{NaN}_{3}$, $0.075 \mathrm{mM}$ DCPIP and $0.1 \mathrm{mM}$ of decylubiquinone. After 2 min of incubation, $0.3 \mathrm{mM}$ NADH was added and the rate of disappearance of DCPIP was monitored at $600 \mathrm{~nm}$. Rotenone $(5 \mu \mathrm{M})$ was added during the measurement to determine the background rate, and the activity was calculated using $\varepsilon$ DCPIP $=19.1 \mathrm{mM}^{-1} \mathrm{~cm}^{-1}$. Specific rotenone-sensitive complex I activity was expressed in $\mathrm{mIU}$ (nanomoles of DCPIP $/ \mathrm{min} / \mathrm{mg}$ protein). Citrate synthase (EC 2.3.3.1) activity was assayed by a standard procedure [14]. Specific enzymatic activities were expressed in mIU, i.e. nanomoles of 5-5'dithiobis (2-nitrobenzoic acid), DTNB/min/mg protein. All experiments were performed in triplicate on independent cell cultures.

\section{Oxygen consumption}

The respiratory rates were measured on cells permeabilized by incubation for $2 \mathrm{~min}$ with digitonin $(15 \mu \mathrm{g} / \mathrm{mil}-$ lion cells) and re-suspended in the respiratory buffer

Table 1 Mitochondrial genetic profile of the LHON fibroblasts

\begin{tabular}{|c|c|c|c|c|c|}
\hline PATIENTS & Age (years) & Sex & mtDNA mutation & Heteroplasmy level & Haplogroup \\
\hline 1 & 22 & $M$ & $m .3460 G>A$ & $100 \%$ & $J$ \\
\hline 2 & 30 & M & m.11778G > A & $74 \%$ & V \\
\hline $3^{a}$ & 43 & $\mathrm{~F}$ & $\mathrm{~m} .11778 \mathrm{G}>\mathrm{A}$ & $100 \%$ & U \\
\hline 4 & 20 & M & m.11778G > A & $>90 \%$ & K \\
\hline 5 & 47 & $M$ & $\mathrm{~m} .11778 \mathrm{G}>\mathrm{A}$ & $100 \%$ & $\mathrm{H}$ \\
\hline 6 & 32 & M & $m .11778 G>A$ & $100 \%$ & V \\
\hline 7 & 44 & $M$ & $\mathrm{~m} .11778 \mathrm{G}>\mathrm{A}$ & $81 \%$ & $J$ \\
\hline 8 & 52 & $M$ & $\mathrm{~m} .11778 \mathrm{G}>\mathrm{A}$ & $100 \%$ & $\mathrm{H}$ \\
\hline 9 & 43 & $M$ & m.14484T > C & $100 \%$ & $H$ \\
\hline
\end{tabular}

although Patient 3 had a typical LHON clinical presentation, she was also known to be carrying an OPA1 gene mutation (p.lle382Met). However, the pathogenicity of this mutation has not been fully demonstrated and its contribution to the clinical expression of LHON in this patient is unknown. 


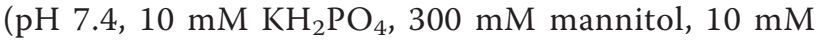
$\mathrm{KCl}$, and $5 \mathrm{mM} \mathrm{MgCl}_{2}$ ). The respiratory rates of 3-5 $\times$ $10^{6}$ cells were recorded at $37^{\circ} \mathrm{C}$ in 2 -ml glass chambers using a high-resolution Oxygraph respirometer (Oroboros, Innsbruck, Austria). Respiration was started with complex I-dependent substrates $(5 \mathrm{mM}$ malate/5 $\mathrm{mM}$ pyruvate). Complex I-coupled state 3 respiration was measured by adding $0.5 \mathrm{mM} \mathrm{NAD}^{+} / 1.5 \mathrm{mM}$ ADP. Then, $10 \mathrm{mM}$ succinate were added to reach maximal coupled respiration, and $10 \mu \mathrm{M}$ rotenone were injected to obtain the complex II-coupled state 3 respiration. Oligomycin $(8 \mu \mathrm{g} / \mathrm{mL})$ was added to determine the uncoupled state 4 respiration. Finally, FCCP $(1 \mu \mathrm{M})$ was added to control the permeabilisation of the fibroblasts. All experiments were performed in duplicate on independent cell cultures.

\section{Statistical analysis}

Vehicle-treated and idebenone-treated fibroblasts from LHON patients and controls were compared using a non-parametric Mann-Whitney $U$ test; differences were considered significant at $p<0.05$.

\section{Results}

Idebenone at $10 \mu \mathrm{M}$ partially restores complex I enzymatic activity

The activity of NADH ubiquinone oxidoreductase was lower in fibroblasts from LHON patients as compared to controls $(56.7 \pm 10.5$ in LHON fibroblasts versus $108.4 \pm 7.4$ in controls; $p=0.01$ ) (Figure 1A). Idebenone at $10 \mu \mathrm{M}$ induced variable increases in complex I enzymatic activity in fibroblasts from LHON patients, whereas it had no such effect on control fibroblasts (Figure $1 \mathrm{~A}$ ). The pooled results of fibroblasts from LHON patients treated with idebenone showed a $42 \%$ increase in complex I enzymatic activity $(81.0 \pm 15.0$ in fibroblasts from LHON patients treated with idebenone versus $56.7 \pm 10.5$ in vehicle-treated fibroblasts; $p=0.002$ ) (Figure 1B).

\section{Idebenone at $10 \mu \mathrm{M}$ induces contradictory effects on respiratory chain activity}

The activity of the mitochondrial respiratory chain was analysed by polarographic measurements of complex Idriven respiration. The I/(I+II) ratio indicated the fraction of complex I-driven respiration with respect to the maximal respiratory rate controlled by complexes I and II. The $\mathrm{I} /(\mathrm{I}+\mathrm{II})$ ratio was significantly lower in fibroblasts from six LHON patients compared to controls $(0.50 \pm$ 0.05 versus $0.72 \pm 0.08 ; p=0.02$ ) (Figure $2 \mathrm{~A}$ ). Idebenone had no effect on complex I-driven respiration in controls. However, in fibroblasts from LHON patients the response to idebenone proved to be contradictory. Thus, in fibroblasts from Patients 1, 3 and 6, complex I-driven respiration was impaired, whereas it was stimulated in fibroblasts from Patients 2, 4 and 5 (Figure 2B). The analysis of these two groups of fibroblasts revealed a deleterious effect of idebenone in the first group, with a $42 \%$ reduction in complex I-driven respiration compared to controls $(p=0.04)$, and a stimulating effect of idebenone in the second group, with a $16 \%$ increase compared to controls $(p=0.04)$ (Figure $2 \mathrm{C}$ ).

\section{Discussion and conclusions}

Previous studies of LHON fibroblasts have shown significant complex I impairment $[3,15]$. These results attest that, although the clinical expression is mostly limited to retinal ganglion cells, the OXPHOS defect is probably more generalized as has also been shown by Barbiroli et al. [16] who found defective energy metabolism in the muscle and brain of LHON patients by using 31P-MRS in vivo. Thus, fibroblasts represent an interesting model to explore the LHON-associated OXPHOS defect and to research molecules able to compensate the defect.

This study demonstrates the impact of idebenone on respiratory chain activity in fibroblasts from nine LHON patients. Indeed, idebenone increased complex I enzymatic activity in these fibroblasts by $42 \%$ compared to controls $(p=0.002)$. This idebenone effect on complex I enzymatic activity was found to be shared by fibroblasts carrying the same m.11778 G > A mutation in 7 different genetic backgrounds (inter-individual variability); this idebenone effect was also found to be shared by fibroblasts carrying the 3 main LHON mutations (intermutation variability). The action of idebenone was not due to an increase either of complex I or of the mitochondrial mass. Indeed, two subunits of complex I, i.e. NDUFA9 and NDUFB8 in western blot, and the citrate synthase activity were not affected by the treatment (Additional file 1: Figure S1).

Various types of action have been ascribed to idebenone in the literature. Experiments with idebenone have led to some conflicting results. Thus, the oral administration of idebenone for 3 days has been reported to stimulate mitochondrial respiration linked to complexes I and II in the rat brain [11]. In contrast, idebenone has been described as a weak substrate for complex I activity $[17,18]$. Idebenone has also been shown to bind to a rotenone-insensitive site, i.e. the non-physiological ironsulphur N2 site, thus leading to higher NADH oxidation but without complete reduction [19]. However, in these latter observations, idebenone was added in the incubation medium directly only during the experiments. The administration of idebenone over a period of 3 days [11] may have led to better metabolization of the drug, allowing it to enter cells and activate signalling pathways such as MAP kinase [10]. 


\section{A}

\section{Complex I activity}

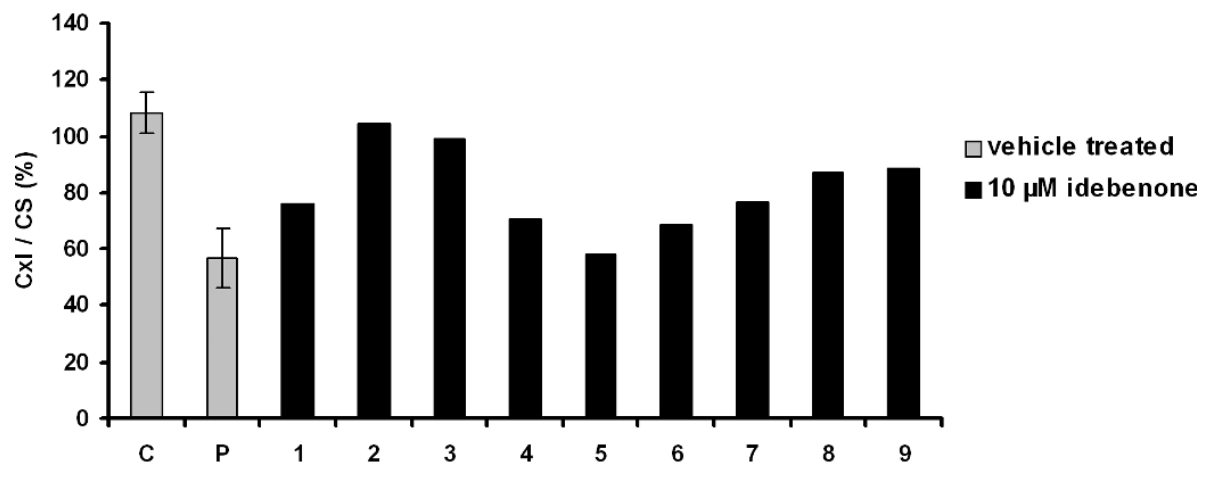

B

Complex I activity

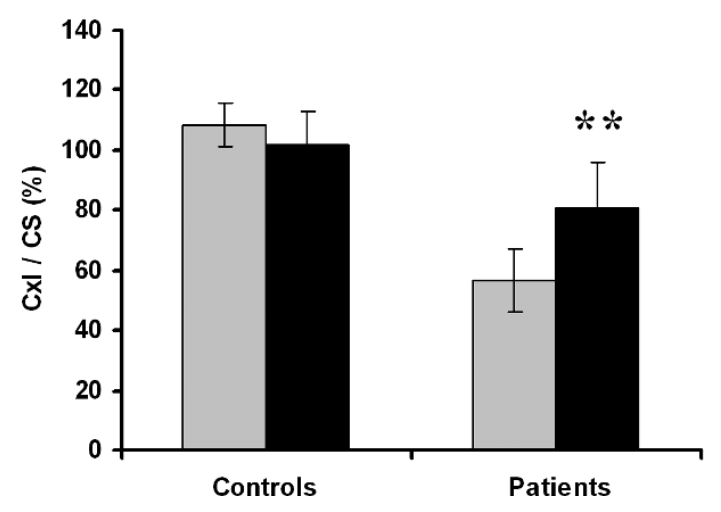

$\square$ vehicle treated

$\square 10 \mu \mathrm{M}$ idebenone

Figure 1 The effect of idebenone on complex I activity. A. Complex I (CxI) activity in fibroblasts from LHON patients ( $\mathrm{n}=9$ ) and controls ( $\mathrm{n}$ =3) treated with vehicle (grey bars) or $10 \mu \mathrm{M}$ idebenone (black bars) $24 \mathrm{~h}$ before the analysis. The enzymatic activity was standardized in terms of citrate synthase (CS) activity, indicative of mitochondrial mass and expressed as the percentage of activity of one control fibroblast used as reference in all experiments $(\mathrm{CxI} / \mathrm{CS}(\%))$. B. Pooled results from fibroblasts from LHON patients and controls. Results are expressed as mean values \pm SD. Statistical significance: ${ }^{* *} p<0.01$ compared with vehicle-treated fibroblasts from LHON patients. $C=C$ Controls and $P=$ Patients.

In our study, the polarographic investigation of the effect of idebenone on mitochondrial activity in fibroblasts from six LHON patients led to contradictory results: a $16 \%$ increase in complex I-driven respiration in three cases, and a $42 \%$ decrease in the other three cases. The increase observed might correspond to the idebenone-induced improvement similar to that reported in intact rat brain mitochondria [11], whereas the opposite response might be explained by an increase of electron escape du to enhanced idebenone fixation at a non-specific site [19].

The variability of the effect of idebenone on complex I activity may be linked to the genetic variability of the LHON patients. However, the number of patients in our study was too small to reveal the possible influence of the haplogroup, the mutation type or the mutation level on the treatment. A larger cohort of LHON patients would be necessary to assess the influence of these factors.

To our knowledge, this is the first demonstration that idebenone directly influences mitochondrial respiratory chain activity in LHON patients. Idebenone increases the enzymatic activity of complex I and acts on the complex I-driven respiration rate, modifying the balance between the electron fluxes provided by complexes I and II. It is tempting to speculate that the variability of the biological response to idebenone in fibroblasts from LHON patients may explain the discordant results observed in some clinical studies $[6,7,20]$. Our results suggest that only a fraction of LHON patients may benefit from the improved respiration afforded by idebenone. If this finding is 

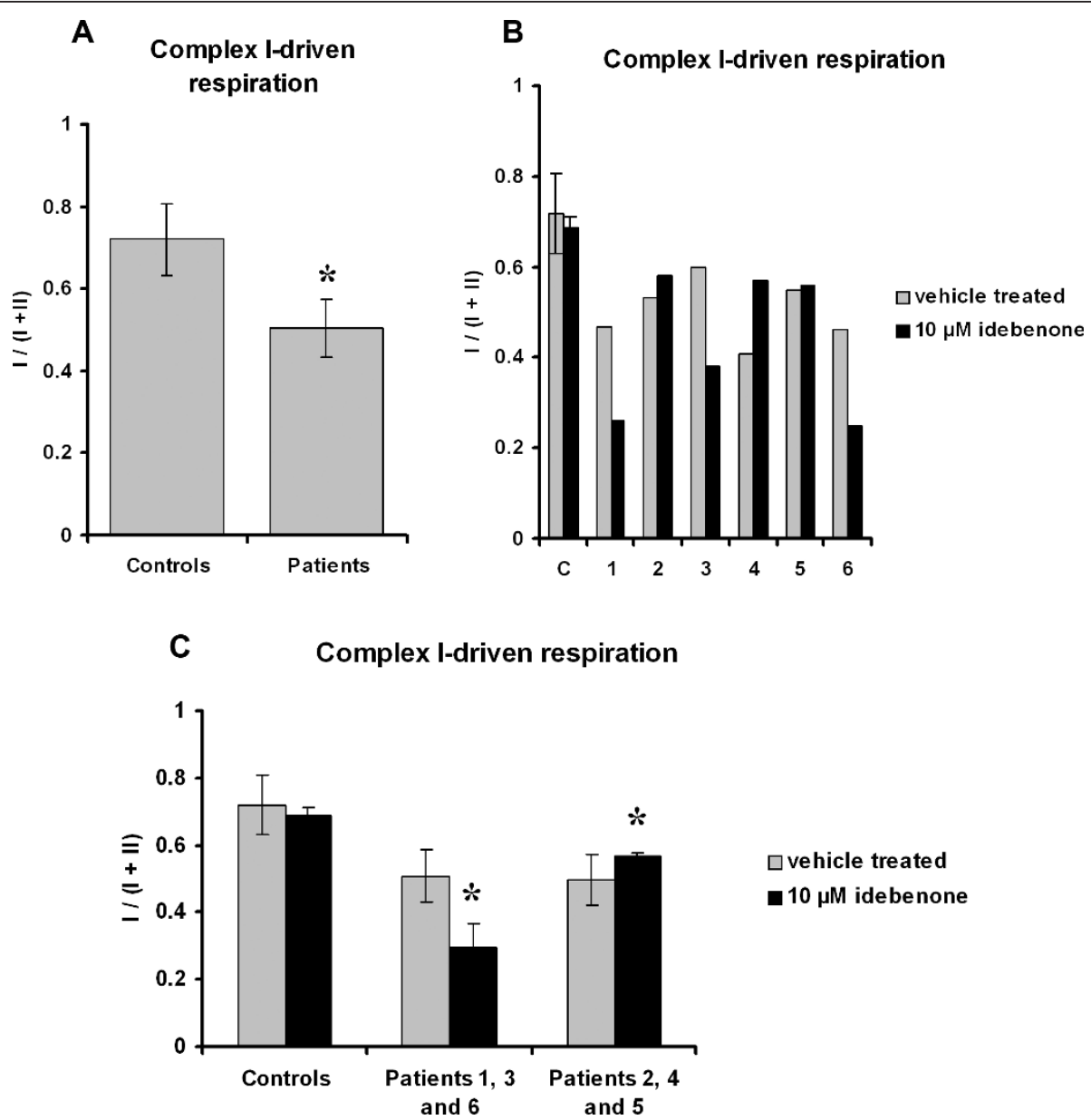

Figure 2 The effect of idebenone on complex I-driven respiration. Determination of complex I-driven respiration in permeabilized fibroblasts from LHON patients $(n=6)$ and controls $(n=3)$ treated with vehicle (grey bars) or $10 \mu M$ idebenone (black bars). The $1 /(I+I)$ ratio corresponds to the fraction of respiration driven by complex I with respect to the maximal respiratory rate driven by complexes I and II. A. Pooled results of fibroblasts from Patients 1-6 and controls. B. Individual results of Patients 1-6 and controls. C. Pooled results of fibroblasts from Patients 1, 3 and 6, in which idebenone impaired respiration, and Patients 2, 4 and 5, in which idebenone stimulated respiration. Results are expressed as mean values \pm SD. Statistical significance: ${ }^{*} P<0.05$ compared with vehicle-treated fibroblasts from LHON patients. $C=$ Controls and $P=P a t i e n t s$.

substantiated by a more extensive study, a preliminary investigation of the impact of idebenone on fibroblasts from LHON patients may be useful to select potentially "good responders" to the treatment.

\section{Availability of supporting data}

The data sets supporting the results of this article are included within the article and its additional file.

\section{Additional material}

Additional file 1: Figure S1. The effect of idebenone on complex I quantity and mitochondrial mass. A. Quantity of two subunits of complex I, NDUFA9 and NDUFB8, in fibroblasts from LHON and controls treated with vehicle $(V)$ or $10 \mu \mathrm{M}$ idebenone (110) 24 hours before the analysis. B. Citrate synthase activity in fibroblasts from LHON patients (n $=9$ ) and controls $(n=3)$ treated with vehicle (grey bars) or $10 \mu \mathrm{M}$ idebenone (black bars) 24 hours before the analysis. The enzymatic activity was expressed as the percentage of activity of one control fibroblast used as reference in all experiments.

\section{Abbreviations}

CS: Citrate synthase; DCPIP: Dichlorophenolindophenol; LHON: Leber's hereditary optic neuropathy; mtDNA: Mitochondrial DNA; ND: NADH dehydrogenase; NUR: NADH ubiquinone reductase; OMIM: Online Mendelian Inheritance in Man; OPA1: Optic atrophy protein 1; OXPHOS: Oxidative phosphorylation.

\section{Acknowledgements}

We are grateful to Stéphanie Chupin and Céline Wetterwald for technical assistance, to Santhera Pharmaceuticals (Switzerland) Ltd for kindly providing idebenone, and to Pr Kanaya Malkani for critical reading and comments on the manuscript. This work was supported by grants from the following research foundations and institutions: "Union Nationale des Aveugles et Déficients Visuels"; "Retina France"; "Ouvrir les Yeux"; "Associations contre les Maladies Mitochondriales"; "Fondation pour la Recherche Médicale" (THERAMIT project); "Agence Nationale de la Recherche" (ERMION E-Rare 
project); and "Union des Industries et Métiers de la Métallurgie de Maine-etLoire".

\section{Author details}

${ }^{1}$ INSERM U771, Angers 49000, France. ${ }^{2}$ CNRS 6214, Angers 49000, France. ${ }^{3}$ LUNAM Université, Angers 49000, France. ${ }^{4}$ Département de Biochimie et Génétique, Centre Hospitalier Universitaire, Angers 49000, France.

${ }^{5}$ Département de Neurologie, Centre Hospitalier Universitaire, Angers 49000 France. ${ }^{6}$ Département d'Ophtalmologie, Centre Hospitalier Universitaire, Angers 49000, France. 'Département de Biochimie et Génétique, CHU d'Angers, 49933 Angers, France.

\section{Authors' contributions}

CA conceived and designed the study, performed literature search, conducted experiment, analyzed and interpreted data, wrote and critically revised the manuscript. NG, VDD and AC participated in all stage of study design, data interpretation and critical revision of the manuscript. VG, CV, JC, MF, DM, PAB, DB, VP participated to the clinical and the biological diagnosis of the patients, and critically revised the manuscript. PR and DL conceived and designed the study, provided direction and oversight of the experiments and critically revised the manuscript. All authors have read and approved the final manuscript.

\section{Competing interests}

The authors declare that they have no competing interests.

Received: 29 August 2011 Accepted: 22 December 2011 Published: 22 December 2011

\section{References}

1. Carelli V, La Morgia C, lommarini L, Carroccia R, Mattiazzi M, Sangiorgi S, Farne S, Maresca A, Foscarini B, Lanzi L, et al: Mitochondrial optic neuropathies: how two genomes may kill the same cell type? Biosci Rep 2007, 27(1-3):173-184.

2. Yu-Wai-Man P, Griffiths PG, Hudson G, Chinnery PF: Inherited mitochondrial optic neuropathies. J Med Genet 2009, 46(3):145-158.

3. Chevrollier A, Guillet V, Loiseau D, Gueguen N, de Crescenzo MA, Verny C, Ferre M, Dollfus H, Odent S, Milea D, et al: Hereditary optic neuropathies share a common mitochondrial coupling defect. Ann Neurol 2008 , 63(6):794-798.

4. Carelli V, Barboni P, Zacchini A, Mancini R, Monari L, Cevoli S, Liguori R, Sensi M, Lugaresi E, Montagna P: Leber's Hereditary Optic Neuropathy (LHON) with 14484/ND6 mutation in a North African patient. J Neurol Sci 1998, 160(2):183-188

5. Mashima Y, Hiida Y, Oguchi Y: Remission of Leber's hereditary optic neuropathy with idebenone. Lancet 1992, 340(8815):368-369.

6. Mashima Y, Kigasawa K, Wakakura M, Oguchi Y: Do idebenone and vitamin therapy shorten the time to achieve visual recovery in Leber hereditary optic neuropathy? J Neuroophthalmol 2000, 20(3):166-170.

7. Barnils N, Mesa E, Munoz S, Ferrer-Artola A, Arruga J: Response to idebenone and multivitamin therapy in Leber's hereditary optic neuropathy. Arch Soc Esp Oftalmol 2007, 82(6):377-380.

8. Klopstock T, Yu-Wai-Man P, Dimitriadis K, Rouleau J, Heck S, Bailie M, Atawan A, Chattopadhyay S, Schubert M, Garip A, et al: A randomized placebo-controlled trial of idebenone in Leber's hereditary optic neuropathy. Brain 2011, 134:2677-2686.

9. Carelli V, La Morgia C, Valentino ML, Rizzo G, Carbonelli M, De Negri AM, Sadun F, Carta A, Guerriero S, Simonelli F, et al: Idebenone Treatment In Leber's Hereditary Optic Neuropathy. Brain 2011, 134:e188.

10. Takuma K, Yoshida T, Lee E, Mori K, Kishi T, Baba A, Matsuda T: CV-2619 protects cultured astrocytes against reperfusion injury via nerve growth factor production. Eur J Pharmacol 2000, 406(3):333-339.

11. Sugiyama Y, Fujita T: Stimulation of the respiratory and phosphorylating activities in rat brain mitochondria by idebenone (CV-2619), a new agent improving cerebral metabolism. FEBS Lett 1985, 184(1):48-51.

12. Loiseau D, Chevrollier A, Verny C, Guillet V, Gueguen N, Pou de Crescenzo MA, Ferre M, Malinge MC, Guichet A, Nicolas G, et al: Mitochondrial coupling defect in Charcot-Marie-Tooth type 2A disease. Ann Neurol 2007, 61(4):315-323.

13. Benit $P$, Slama A, Rustin P: Decylubiquinol impedes mitochondrial respiratory chain complex I activity. Mol Cell Biochem 2008, 314(1-2):45-50.
14. Schulman JD, Blass JP: Measurement of citrate synthase activity in human fibroblasts. Clin Chim Acta 1971, 33(2):467-469.

15. Bonnet C, Augustin S, Ellouze S, Benit P, Bouaita A, Rustin P, Sahel JA, Corral-Debrinski M: The optimized allotopic expression of ND1 or ND4 genes restores respiratory chain complex I activity in fibroblasts harboring mutations in these genes. Biochim Biophys Acta 2008, 1783(10):1707-1717.

16. Barbiroli B, Montagna P, Cortelli P, lotti S, Lodi R, Barboni P, Monari L, Lugaresi E, Frassineti C, Zaniol P: Defective brain and muscle energy metabolism shown by in vivo 31P magnetic resonance spectroscopy in nonaffected carriers of 11778 mtDNA mutation. Neurology 1995, 45(7):1364-1369

17. Lenaz G, Bovina C, D'Aurelio M, Fato R, Formiggini G, Genova ML, Giuliano G, Merlo Pich M, Paolucci U, Parenti Castelli G, et al: Role of mitochondria in oxidative stress and aging. Ann N Y Acad Sci 2002, 959:199-213.

18. Esposti MD, Ngo A, Ghelli A, Benelli B, Carelli V, McLennan H, Linnane AW: The interaction of $\mathrm{Q}$ analogs, particularly hydroxydecyl benzoquinone (idebenone), with the respiratory complexes of heart mitochondria. Arch Biochem Biophys 1996, 330(2):395-400.

19. Fato R, Bergamini C, Leoni S, Lenaz G: Mitochondrial production of reactive oxygen species: role of complex I and quinone analogues. Biofactors 2008, 32(1-4):31-39.

20. Yu-Wai-Man P, Griffiths PG, Chinnery PF: Mitochondrial optic neuropathies-disease mechanisms and therapeutic strategies. Prog Retin Eye Res 2011, 30(2):81-114.

doi:10.1186/1756-0500-4-557

Cite this article as: Angebault et al:: Idebenone increases mitochondrial complex I activity in fibroblasts from LHON patients while producing contradictory effects on respiration. BMC Research Notes 2011 4:557.

\section{Submit your next manuscript to BioMed Central and take full advantage of:}

- Convenient online submission

- Thorough peer review

- No space constraints or color figure charges

- Immediate publication on acceptance

- Inclusion in PubMed, CAS, Scopus and Google Scholar

- Research which is freely available for redistribution

Submit your manuscript at www.biomedcentral.com/submit
C) Biomed Central 